\title{
Editorial
}

\section{Editorial: ORCID is a Wonderful (But Not Required) Tool for Authors}

\author{
Seth S. Leopold (i)
}

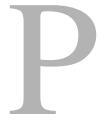
eriods of history have been named for the tools of the day-stone age, stone tools; bronze age, bronze tools. We again live in an era of tools. Future archaeologists, no doubt, will devote some future annual meeting to coming up with a suitable name for our age.

And like our Neolithic forebears, we must decide which tools to adopt, and how to integrate them into our busy lives. While swinging a stone hammer beat not having one every time, our choices today seem less clear. We often hear of new websites

The author certifies that he, or any members of his immediate family, has no commercial associations (eg, consultancies, stock ownership, equity interest, patent/licensing arrangements, etc) that might pose a conflict of interest in connection with the submitted article.

All ICMJE Conflict of Interest Forms for authors and Clinical Orthopaedics and Related Research ${ }^{\circledR}$ editors and board members are on file with the publication and can be viewed on request.

The opinions expressed are those of the writers, and do not reflect the opinion or policy of $C O R R^{\circledR}$ or the Association of Bone and Joint Surgeons ${ }^{\circledR}$.

S. S. Leopold $(\square)$

Clinical Orthopaedics and Related

Research, Philadelphia, PA 19103, USA

e-mail: sleopold@clinorthop.org offering to help academic orthopaedic surgeons and musculoskeletal scientists track, promote, or disseminate their work. And like our prehistoric cousins, we are forced by time and evolution to decide whether to adopt new tools or risk extinction.

Lately one new tool, ORCID (Open Researcher and Contributor ID), has garnered much attention. Even though ORCID has been available for several years, a surprising number of orthopaedic researchers and readers still do not know about it. In short summary, ORCID is an international nonprofit effort to provide every researcher with a unique identification number, (which ORCID calls an "iD”). While ORCID is not the first player in the sphere of author identification, it seems to have become the dominant one. In partnership with universities, publishers, and societies, as of January 2016 ORCID has issued nearly 2 million iDs [5]. The green icon in my byline above means I have one of them.

ORCID's chief benefit is disambiguation. While each of us is unique, most of our names are not, and this can make it difficult for universities, grant funding agencies, and others to link us correctly to our publications. For example, as of this writing, the mostcommon names in the United States and China (J Smith and W Zhang) were linked to 22,257 and 26,279 manuscripts, respectively, on PubMed. That's quite a CV.

ORCID seeks to solve this problem by allowing each author who signs up to receive a unique identifier at no charge. My ORCID iD allows www.ORCID.org to separate my work from that of my PubMed doppelgänger, another SS Leopold, who writes mainly on matters of the heart (and lungs). As my work gets published, ORCID updates an online bibliography that is authentically mine, which I can choose to share with institutions or funding agencies, or which I can keep private. Departments find this facilitates tracking faculty productivity (indeed some universities create ORCID iDs for all faculty, in part for this reason [4]), it can make grant submission easier, and future uses like digital repositories and more-efficient approaches to data sharing may improve life both for clinician-scientists and the patients whom they care for. Authors whose names have changed during the course of their careers may find ORCID especially handy, since it links to an individual's entire oeuvre regardless of name changes.

Because of these benefits, a number of journals, societies, and publishers 


\section{Editorial}

have decided to make ORCID a requirement to publish work with them [6]. Some of these are among the largest names in the world of scholarly publishing-including the journals Science and Public Library of Science, as well as the Royal Society, the major scientific academy in the United Kingdom.

CORR $^{\circledR}$ will not be joining them in requiring the use of ORCID, at least not now.

Why not? The major benefit to a journal, as noted, is disambiguation. Unfortunately, notwithstanding the last-two letters of its acronym, ORCID does not reliably identify that an author is who he or she claims to be. Even though it is not marketed as a validation tool, the self-described "registry of unique researcher identifiers" lends an air of confirmation or substantiation of an author's identity when an ORCID iD is attached to a published manuscript. As a colleague stated in response to some enthusiastic promotion of ORCID on a scholarlypublishing blog [3], "The problem is not that ORCID does not validate identification, the problem is that it is not obvious that ORCID does not validate identification." Stated otherwise, ORCID provides disambiguation where authors who play fair are concerned, but it is easily subverted by authors who do not work according to commonly held standards of authorship responsibility [1]. In such situations, ORCID can do little more than to provide the appearance of disambiguation. This is potentially misleading to readers and to journals, which need to be able to reach a responsible author if a manuscript's integrity is questioned.

In Europe and the United States, the idea that someone would share an ORCID iD-such that when one writes a paper, another gets credit-seems incongruous. But in some cultures, online identity sharing is more commonplace. Interviews we have conducted with authors and editors revealed that in China, younger faculty members routinely provide ghost authorship for more-senior "authors" to gain professional advancement. Several authors may share one email address, and individual authors submit work from a number of different email addresses. The latter may be a practical solution to inconsistent email service in many parts of China. However, the former represents a serious problem for journals that wish to have authors uphold the responsibilities of authorship as articulated by the International Committee of Medical Journal Editors [2] and others. Ghost authorship is not a problem limited to China by any means, but thus far, the online identity sharing we have observed has been.

The identity-sharing problem is further exacerbated when multiple authors use the same ORCID iD, which we also have seen and reported to ORCID, which as of now has no formal mechanism to prevent this kind of fraud. We believe that any system based on an individual asserting his or her identity by email alone is vulnerable, both to fraud and to hacking. ORCID obviously did not create this problem, but neither can editors nor grant-funding agencies can assume that requiring authors to use ORCID will stop it.

When an author signs in to Editorial Manager ${ }^{\circledR}$ to submit a manuscript to $\operatorname{CORR}^{\circledR}$, he or she has the opportunity to do so using an ORCID iD. By doing so, the manuscript, if published, will automatically be entered into the ORCID database under that author's name. When that occurs, the little green icon (like the one above) will be printed with the author's name on the manuscript, and provide a link to the information that author shares on ORCID.

Some authors will find ORCID to be a useful tool; many institutions already do. Our authors who find it helpful should feel free to use ORCID when they send work to $C O R R^{\circledR}$, but $C O R R^{\circledR}$ will not require authors to use ORCID.

Acknowledgment The author acknowledges with gratitude Lee Beadling BA, for his thoughtful suggestions. 


\section{Editorial}

\section{References}

1. Council of Science Editors. Authorship and authorship responsibili ties. Available at http://www.councilscienceeditors.org/resource-library/ editorial-policies/white-paper-on-publication-ethics/2-2-authorship-and-authorship-responsibilities/. Accessed on February 12, 2016.

2. International Committee of Medical Journal Editors. Defining the role of authors and contributors. Available at: http://www.icmje.org/recommendations/browse/roles-and-responsibiliti es/defining-the-role-of-authors-andcontributors.html. Accessed February 8, 2016.

3. Meadows A. Why some publishers are requiring ORCID iDs for authors: An Interview with Stuart Taylor, The Royal Society. The Scholarly Kitchen. Available at http://scholarlykitchen.ssp net.org/2016/01/07/why-some-publishers-are-requiring-orcid-ids-for-authorsan-interview-with-stuart-taylor-the-ro yal-society/. Accessed on February 8, 2016.

4. MIT Libraries. ORCID \& author identifiers: Link your name \& your research: Home. Available at http:// libguides.mit.edu/authorids. Accessed on February 8, 2016.

5. ORCID. Number of ORCID iDs. Available at http://support.orcid.org/ knowledgebase/articles/150557-number-of-orcid-ids. Accessed February 8, 2016.

6. ORCID. Requiring ORCID in publication workflows: Open letter. Available at https://orcid.org/content/requiring-orcid-publication-workflowsopen-letter. Accessed February 12, 2016. 